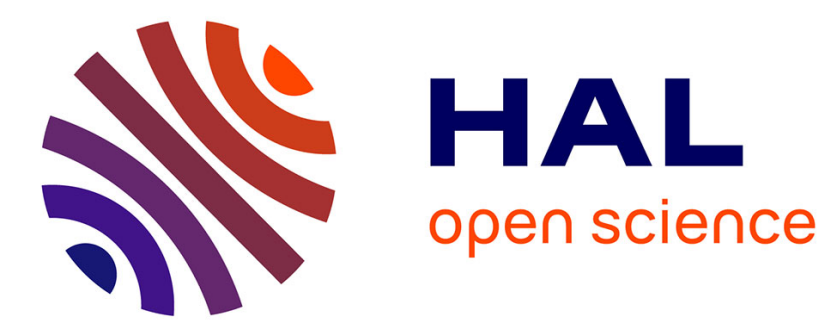

\title{
A flexible BIST strategy for SDR transmitters
}

\author{
Emanuel Dogaru, Filipe Vinci dos Santos, William Rebernak
}

\section{To cite this version:}

Emanuel Dogaru, Filipe Vinci dos Santos, William Rebernak. A flexible BIST strategy for SDR transmitters. 2014 Design, Automation \& Test in Europe Conference \& Exhibition (DATE), Mar 2014, Dresden, Germany. pp.1 - 6, 10.7873/DATE2014.383 . hal-01005401

\section{HAL Id: hal-01005401 \\ https://hal-centralesupelec.archives-ouvertes.fr/hal-01005401}

Submitted on 12 Jun 2014

HAL is a multi-disciplinary open access archive for the deposit and dissemination of scientific research documents, whether they are published or not. The documents may come from teaching and research institutions in France or abroad, or from public or private research centers.
L'archive ouverte pluridisciplinaire HAL, est destinée au dépôt et à la diffusion de documents scientifiques de niveau recherche, publiés ou non, émanant des établissements d'enseignement et de recherche français ou étrangers, des laboratoires publics ou privés. 


\title{
LMS-Based RF BIST Architecture for Multistandard Transmitters
}

\author{
Emanuel Dogaru* ${ }^{* \dagger}$, Filipe Vinci dos Santos* and William Rebernak ${ }^{\dagger}$ \\ *Thales Chair on Advanced Analog Design, SUPELEC \\ Gif-sur-Yvette, France \\ Email: filipe.vinci@supelec.fr \\ ${ }^{\dagger}$ THALES Communications \& Security \\ Gennevilliers, France
}

\begin{abstract}
Software defined radios (SDR) platforms are increasingly complex systems which combine great flexibility and high performance. These two characteristics, together with highly integrated architectures make production test a challenging task. In this paper, we introduce a Radio Frequency (RF) Builtin Self-Test (BIST) strategy based on Periodically Nonuniform Sampling of the signal at the output stages of multistandard radios. We leverage the I/Q ADC channels and the DSP resources to extract the bandpass waveform at the output of the power amplifier (PA). Analytical expressions and simulations show that our time-interleaved conversion scheme is sensitive to time-skew. We propose a time-skew estimation technique based on a Least Mean Squares (LMS) algorithm to solve this problem. Simulation results show that we can effectively reconstruct the bandpass signal of the output stage using this architecture, opening the way for a complete RF BIST strategy for multistandard radios.
\end{abstract}

Keywords-BIST, Periodically nonuniform sampling, software radios, mixed-signal test, undersampling, spectral mask estimation, LMS calibration, time-skew estimation

\section{INTRODUCTION}

The Software Defined Radio concept proposed by Mitola [1] is a Radio in which some (or all) of the physical layer functions are programmable. The outstanding flexibility and performance of modern SDRs result from careful trade-offs among advanced analog/RF circuitry, high-speed reconfigurable digital hardware and sophisticated real-time software. However, the inherent adaptability of these multistandard platforms hinders the testability of the finished units. Unlike conventional transmitters, SDR platforms must satisfy strict requirements under a wide variety of operating modes. Established mixed-signal and RF test strategy are either too time-consuming (thus costly) or can't ensure compliance with several modulations standards, including those yet to appear.

Over the past few years, analog and mixed-signal test and testability has been a subject of intense research. Traditional production testing of transceivers relies on specialized machines, known as Automatic Test Equipment (ATE) units, [2], [3]. ATEs are fast, accurate and reconfigurable, but also very expensive and hard to master. A cost effective alternative to the ATEs is the idea of introducing Built-In Self-Test (BIST) techniques to eliminate or reduce the need for external instrumentation. Analog and mixed-signal (AMS) BIST techniques generally entail additional circuitry and reuse of resources available (DSP, converters, memory).
For the RF transceivers, the loopback approach is one of the most cited RF BIST technique (see [4]-[7]). In a nutshell, RF loopback consists in using the transmitting part (Tx) to excite some parts or all of the receiver ( $\mathrm{Rx})$. The key feature is to add components that allow the reconfiguration of the on-board (or on-chip) resources to carry out some type of characterization.

Loopback BIST is an attractive proposition, although it has one major drawback: fault masking. The fault masking is a situation where a (non-catastrophic) failure of the Tx is covered up by an exceptionally good Rx, or the inverse. A marginal product could then go undetected (test escapes). In spite of its shortcomings, loopback BIST has been often implemented and reported as effective [4]-[7].

The aim of our work is to reduce post-manufacture test cost of SDR transceivers by leveraging the reconfigurability of the platform. We are exploring the use of nonuniform sampling (undersampling) to carry out bandpass conversion of the output signal, using I/Q channel ADCs as a single time-interleaved ADCs (see Fig. 1). Our initial efforts are focused to the characterization of the transmitter (Tx) chain with respect to compliance to the spectral mask. The bandpass measurements of the output stage waveforms using nonuniform sampling allow us to address the most vexing post-manufacture test issue for tactical radio units. Our approach is scalable across a large set of complex specifications without incurring additional hardware or performance cost. As presented in in this paper, the technique we propose is more suitable for an offline implementation.

This paper gives a detailed description of our bandpass nonuniform sampling architecture. First, we describe the mathematical basis for the technique, and give theoretical results suggesting its feasibility. We show then that most implementation requirements can be met with reasonable margin without affecting the robustness of signal reconstruction. The most critical requirement is shown to be the time-skew between channels. The feasibility of using time-skew measurement techniques commonly applied in Time-Interleaved ADCs (TIADCs) is analyzed, and experimentally demonstrated with a typical configuration. The results obtained with our simulations are promising, pushing us to advance to a hardware implementation.

To confirm the results of our mathematical analysis we built a behavioral model of an homodyne transmitter and ran extensive simulations in Matlab. Our simulations show that 


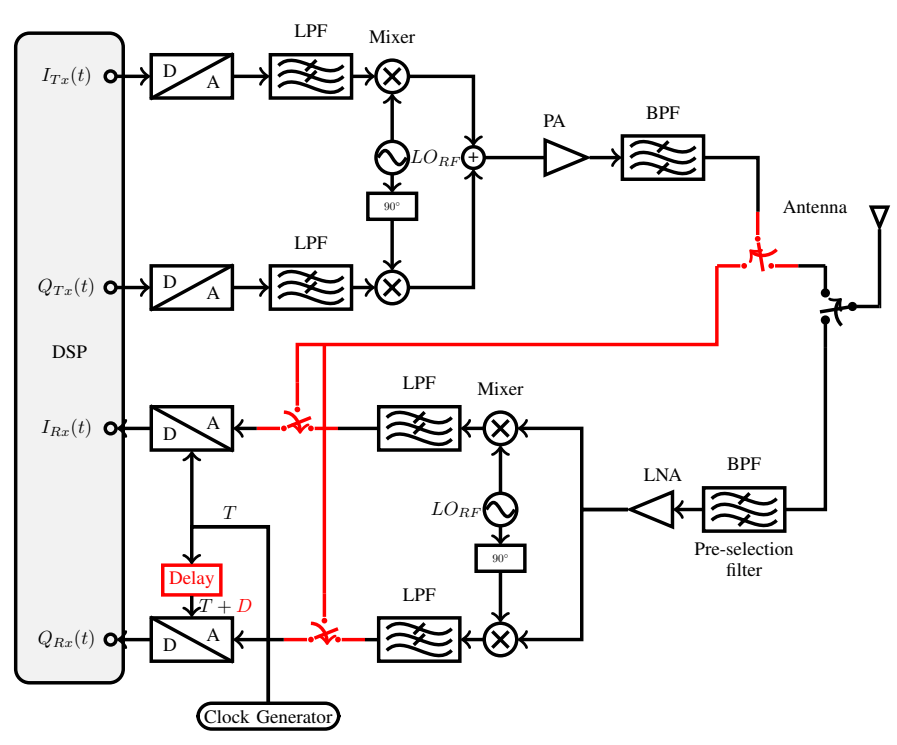

Fig. 1. Architecture of a homodyne transceiver. The red blocks are the modifications required by the RF BIST technique we propose.

the time-skew is indeed critical to our approach, and that it can be estimated to the required level through several means.

The remainder of this paper is organized as follows. Section II introduces the mathematical theory behind the periodical nonuniform sampling technique and motivates our choice. Section III describes a practical realization of the nonuniform sampler and discusses the obstacles found. Section IV provides a time-skew detection technique that meets the nonuniform sampler requirements. Section V shows the results obtained in simulation. Finally, our conclusions and future developments are given in Section VI.

\section{THEORETICAL BACKGROUND}

It is well known that a continuous time signal $f(t)$ with Fourier transform $F(\nu)$, limited to a non-zero frequency range $|\nu|<B$ can be reconstructed from its samples $f(n T / 2)$, where $T=1 / B$. This technique is commonly called Nyquist sampling. It has also been demonstrated in [8], [9] that if $F(\nu)$ is limited to a frequency range $f_{l}<|\nu|<f_{l}+B$ (see Fig. 2), then $f(t)$ can also be reconstructed from a set of uniformly spaced samples $f(n T / 2)$, as long as the ratio $f_{l} / B$ is a positive ratio integer. This sampling scheme is called Periodically Bandpass Sampling of First Order (PBS) or Uniform Bandpass Sampling. If the previous requirements of signal band locations are not met, Kohlenberg [9] showed that $f(t)$ can still be reconstructed from two sets of uniform samples $f(n T)$ and $f(n T+D)$. This sampling scheme is called Periodically Nonuniform Bandpass Sampling of Second Order (PNBS), and will be described in more detail further on.

In this section we present a succinct comparison between the two bandpass sampling techniques. We then show the advantages of using nonuniform sampling over uniform sampling for narrowband signals located at high frequency in the context of SDR testing. Afterwards, we will examine some concerns and potential problems arising from practical implementations of a nonuniform sampler.

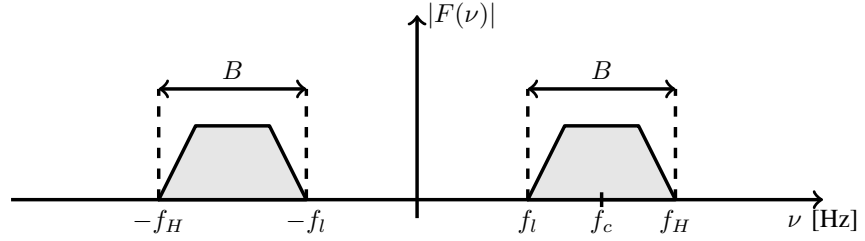

Fig. 2. Bandpass Signal

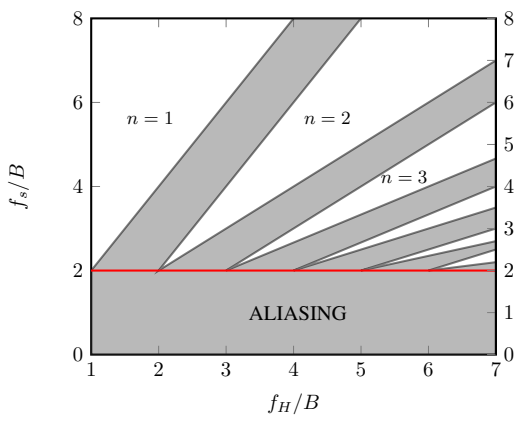

(a)

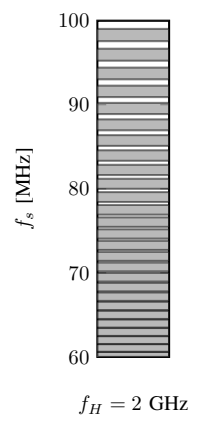

(b)
Fig. 3. The constraints on the sampling rate $f_{s}$ for PBS [12]: (a) the general case and (b) a particular case where $f_{H}=2.03 \mathrm{GHz}$ and $B=30 \mathrm{MHz}$.

\section{A. Periodic Bandpass Sampling}

The advantage of bandpass sampling over Nyquist baseband sampling for RF systems is clear: the sampling frequency needed and the subsequent processing rate are proportional to the information bandwidth, rather than to the center (carrier) frequency.

The periodically uniform sampling technique is commonly used in bandpass sampling receivers [10], [11]. The desired bandpass signal is frequency translated to baseband by the sampler. In other words, the ideal sampling operation creates replicas of the original spectrum in each Nyquist zone. Because of this, a bandpass filter (BPF) is required, otherwise unwanted signals outside the desired bandpass range will also appear at the $1^{\text {st }}$ Nyquist zone (baseband).

The superposition of spectra due to frequency folding (aliasing) is a concern for the choice of sampling frequency $f_{s}$. In effect, the relationship among $f_{s}$, signal bandwidth $B$, and upper range limit $f_{H}$ is constrained if spectral superposition must be avoided. The feasible combinations are depicted graphically in Fig. 3a [12], where $f_{s}$ and $f_{H}$ are normalized w.r.t. signal bandwidth $B$. The white regions are the situations where the bandpass sampling will not result in aliasing. The gray regions represent conditions where alias is occurring. One readily observes that the minimum ideal sampling rate is $f_{s}=2 B$, as expected, but there is little margin for imperfections in the actual implementation. This sensitivity worsens as $f_{H} / B$ increases. Therefore, practical implementations of uniform bandpass sampling must sample faster than the theoretical minimum rate and even then, not all faster rates will avoid aliasing, if the signal band is not well positioned.

Without loss of generality, let's consider a bandpass signal with $B=30 \mathrm{MHz}$ and located at $f_{l}=2 \mathrm{GHz}$. For this example the acceptable sample rates are within the white areas 
in Fig. 3b. If a sampling rate around the ideal minimum value $2 B$ is desired, the subsampling clock should have a precision of few $\mathrm{KHz}$ in order to avoid aliasing. The most obvious solution to relax these constraints is to use guards bands (which is equivalent to sampling at nonminimum rates [12]). Even so, as shown in Fig. $3 \mathrm{~b}$, the sampling around $f_{s}=90 \mathrm{MHz}$ (larger than $2 B$ ) would still require sampling precision of few hundreds of $\mathrm{KHz}$.

To sum up, uniform bandpass sampling is effective, but difficult to implement without incurring aliasing as the ratio $f_{H} / B$ rises.

\section{B. Periodically Nonuniform Bandpass Sampling (PNBS)}

The discussions above suggest that the PBS technique is not well suited for test of software radios due to the lack of flexibility. Indeed, one of the most important characteristics of a software radio is its flexibility. That means that it is capable to operate over a wide range of operating parameters (frequency, data rate, modulation type, etc.). A well designed test strategy should be able to cover all of these configurations, with minimum of effort and extra circuitry. This is hardly the case for a PBS system.

An alternative solution to the PBS is the PNBS technique. PNBS allows the reconstruction of bandpass signals from two sets of uniformly spaced samples, at the theoretical minimal rate and independent of the band locations (the straight red line in Fig. 3). PNBS overcomes the limitations of the PBS and offers a higher level of flexibility.

Bandpass signal reconstruction from nonuniform samples was introduced in [9] with further results given in [8] and [12]. According to [9], a bandpass signal $f(t)$ (Fig. 2) can be reconstructed from two sets of uniform samples, $f(n T)$ and $f(n T+D)$ using the exact interpolation relation:

$$
f(t)=\sum_{n=-\infty}^{+\infty}[f(n T) s(t-n T)+f(n T+D) s(n T+D-t)]
$$

where $T=1 / B, D$ represents the phase delay and:

$$
\begin{aligned}
s(t) & =s_{0}(t)+s_{1}(t) \\
s_{0}(t) & =\frac{\cos \left[2 \pi\left(k B-f_{l}\right) t-k \pi B D\right]-\cos \left[2 \pi f_{l} t-k \pi B D\right]}{2 \pi B t \sin k \pi B D} \\
s_{1}(t) & =\frac{\cos \left[2 \pi\left(f_{l}+B\right) t-k^{+} \pi B D\right]-\cos \left[2 \pi\left(k B-f_{l}\right) t-k^{+} \pi B D\right]}{2 \pi B t \sin k^{+} \pi B D} \\
k & =\left\lceil\frac{2 f_{l}}{B}\right\rceil, k^{+}=k+1, \text { where }\lceil *\rceil \text { is the ceiling operator }
\end{aligned}
$$

Proofs for equations (1), (2) and (3) and further discussions regarding the stability of reconstruction can be found in [9] and [8]. In this paper our concern is the practical realization of a nonuniform sampler within a typical SDR platform. In the following we present some considerations regarding the choice of the delay $D$, that directly affect the architecture of the nonuniform sampler we propose.

1) Choice of $D$ : The relation (1) is valid provided that $D$ meets the following contraints:

$$
\begin{aligned}
& D \neq n T / k \\
& D \neq n T /(k+1), \forall n \in \mathbb{N}
\end{aligned}
$$

If $D$ assumes values which violate the conditions (3) then the reconstruction filter becomes unstable. If $k=\frac{2 f_{l}}{B}$, the first term of the reconstruction filter $s_{0}(t)$ is 0 and the condition ( $\left.3 \mathrm{a}\right)$ no longer applies.

The nonuniform sampling of $f(t)$ can be done by two identical ADCs, both running at the sample rate, $f_{s}$, but triggered with a constant time delay $D$. Intuitively, it is clear that the value assumed by the delay $D$ is critical for the accuracy and computational cost of the signal reconstruction from the nonuniform sampled values. One can observe that if $D$ approaches the right-hand values given in (3), the coefficients of the reconstruction filter rise progressively toward infinity.
Unduly large values complicate the practical realization, since more terms will have to be evaluated with increased accuracy. The authors in [12] showed that the optimal values of $D$ w.r.t. the magnitude of the filter coefficients in (1) are $D= \pm 1 /\left(4 f_{c}\right)$.

2) Reconstruction Robustness: Our aim is to use slow timeinterleaved ADCs to obtain nonuniformly spaced samples of $f(t)$. In order to apply the relation (1), $D$ must be known. Let's say that instead of knowing the real value of $D$, only an estimate $\hat{D}=D+\Delta D$ is available. It has been shown in [12] that the relative difference between the reconstructed spectrum $\hat{F}(\nu)$ and the actual spectrum $F(\nu)$ can be approximated as:

$$
\Delta F=\left|\frac{\hat{F}(\nu)-F(\nu)}{F(\nu)}\right| \approx \pi B(k+1) \Delta D
$$

Equation (4) shows that, as the ratio between the center frequency and the signal bandwidth increases, the acceptable values for $\Delta D$ become very small. Moreover, the precision of reconstruction depends also on the high frequency $f_{c}$, and not only on the signal bandwidth $B$, as it would be desirable. This means that for higher carrier frequency, the estimate of $D$ should be more accurate.

For example, for a bandpass signal at $f_{c}=1 \mathrm{GHz}$ to be recovered from the samples of two ADCs running at $f_{s}=B=$ 
$80 \mathrm{MHz}$ with a precision of $\Delta F=1 \%, \Delta D$ must satisfy:

$$
\Delta D \leq \frac{1}{25} \frac{0.01}{\pi 80 \cdot 10^{6}} \approx 2 \mathrm{ps}
$$

The result above is significant because it shows that a robust bandpass reconstruction with PNBS is feasible. Indeed, there are several hardware implementations reported [13], [14]) that can estimate and correct the time-skew between two ADCs with a granularity of few ps. Finally, equation (4) and the example from (5) establish that the most critical point while reconstructing a bandpass signal using nonuniform sampling is the accurate knowledge of $D$.

3) Remarks: Our main objective is to develop a nonuniform bandpass sampling architecture coupled to a delay identification technique that will overcome the obstacles previously discussed. Using the SDR capabilities and the bandpass nonuniform sampler, our final purpose is to reconstruct and to observe the spectrum at the output of the Tx.

At this stage it is useful to discuss some inherent limitations of our proposed architecture:

- Wideband Noise: Compared to a classic analog receiver that uses mixers to translate the signal to baseband, a bandpass sampling technique degrades the Signal-to-Noise Ratio of the original signal. Even if a bandpass filter is used, the thermal noise is still aliased to the band of interest [11], [12]. For our needs, this limitation is not a concern because we wish to characterize the $\mathrm{Tx}$ at higher signal levels.

- Phase Noise: It is well known that the phase noise of the sampling clock degrades the performance of the bandpass sampling technique [10]. Nonetheless, in [15] the authors showed that the clock jitter requirement for a bandpass sampling receiver are similar to the requirements for the local oscillator (LO) in a mixing receiver.

\section{Proposed Architecture}

The theoretical results presented in the previous section allowed us to derive the general requirements on a nonuniform sampler designed to reconstruct a high-frequency bandpass signal. In this section a possible architecture is described. The architecture we propose is similar to a two-channels timeinterleaved ADC (TIADC). It is shown that time-skew estimation schemes can be adopted by our nonuniform sampling BP-TIADC.

The nonuniform BP-TIADC architecture is shown in Fig. 4. It is only slightly different from a standard two channels TIADC [16]. The key block is the Digitally Controlled Delay Element (DCDE) shown in red. The function of the DCDE is to adjust the delay $D$ such that it meets the constraints (3), as explained in Section II-B.

The objective of our architecture is to implement a nonuniform bandpass sampler at the output stages of an SDR. The receiver side of an SDR platform integrates two powerful ADCs that are idle during transmission. Therefore, we propose to reuse these two ADCs for Tx test purposes. The required modifications are presented in red in Fig. 1. The additional

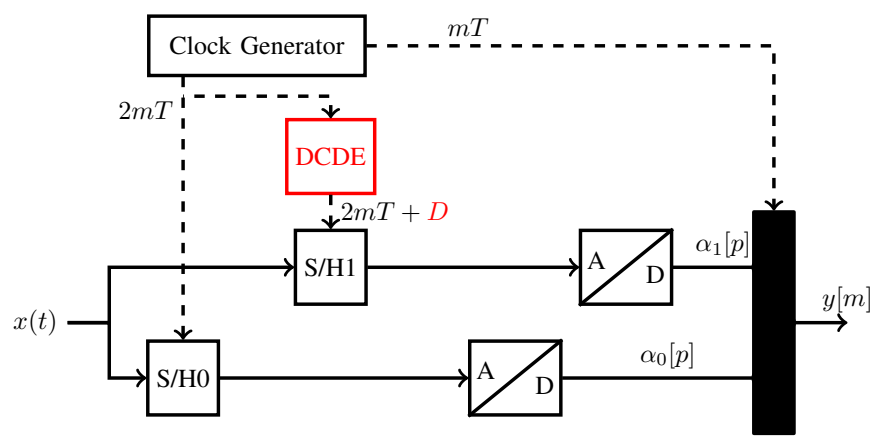

Fig. 4. Block diagram of the proposed BP-TIADC architecture. The key block is the Digitally Controlled Delay Element (DCDE)

hardware required is minimized and the implementation burden is shifted toward the software/digital domain.

The overall reconstruction properties of the nonuniform sampler worsen due to the unavoidable mismatches between the two channels of a manufactured chip. The most important mismatches are offset error, gain error, and time-skew. The offset and the gain error calibrations are relatively simple to implement [16], and will not be discussed further. Time-skew calibration, on the other hand, is a more challenging task. Nonetheless, one should note that the time-skew calibration in standard TIADC architectures is a more difficult endeavor, since one must estimate the delay across channels and then suppress it. The critical effort is this correction, which typically hits the ultimate limits of the underlying technology. This is not the case for the BP-TIADC with nonuniform sampling, where a null (or exact) adjustment of time-skew is not necessary. Our challenge is how to estimate it accurately. The time-skew estimation problem will be discussed in the following section.

\section{Time-Skew Detection Technique}

Several time-skew calibration techniques for TIADCs have been proposed [13], [14]. We have tested the technique proposed by [14] and we have obtained unsatisfactory results. Due to this, we have developed a new LMS-based identification technique designed to accurately estimate the delay $D$ between the two ADCs in the nonuniform sampler.

\section{A. Problem Statement}

Let us denote by $f_{D}^{T}(t)$ the practical reconstruction of $f(t)$ from the samples $f(n T)$ and $f(n T+D)$ :

$f_{D}^{T}(t)=\sum_{n=-n_{w} / 2}^{+n_{w} / 2}[f(n T) s(t-n T)+f(n T+D) s(n T+D-t)]$

where $n_{w}+1$ is the number of taps of the reconstruction filter. Let $\mathbf{t}=\left\{t_{i}, i=\overline{1, N}\right\}$ be a vector of $N$ time values and let $f_{D}^{T}(\mathbf{t})=\left\{f_{D}^{T}\left(t_{i}\right), i=\overline{1, N}\right\}$ be the reconstructed values of $f(\mathbf{t})=\left\{f\left(t_{i}\right), i=\overline{1, N}\right\}$.

The time-skew estimation problem can be defined as the following minimization problem: having an initial estimate $\hat{D}_{0}$, find $\hat{D}$ that minimizes the cost function $\varepsilon_{T_{1}, \hat{D}}^{T, \hat{D}}(\mathbf{t})$ or

$$
\min _{\hat{D}} \varepsilon_{T_{1}, \hat{D}}^{T, \hat{D}}(\mathbf{t})
$$


where the metric

$$
\varepsilon_{T, \hat{D}}^{T, D}(\mathbf{t})=\frac{\sum_{i=1}^{N}\left(f_{\hat{D}}^{T}\left(t_{i}\right)-f_{D}^{T}\left(t_{i}\right)\right)^{2}}{N}
$$

is a measure of how close from real $D$ is the estimate $\hat{D}$.

Let's denote by $m$ the first value of $D$ for which the metric $\varepsilon_{T_{1}, m}^{T, m}(\mathbf{t})$ is undefined: $m=\min \left\{\frac{1}{k^{+} B}, \frac{1}{k_{1}^{+} B_{1}}\right\}, B=$ $1 / T, B_{1}=1 / T_{1}$. It is easy to prove that, for $T<T_{1}$, if the following requirements are met:

$$
\begin{gathered}
k^{+} B \neq k_{1} B_{1} \\
k^{+} B \neq k_{1}^{+} B_{1} \\
D \in] 0, m[
\end{gathered}
$$

then the cost function $\varepsilon_{T_{1}, \hat{D}}^{T, \hat{D}}(\mathbf{t})$ has only one minimum in the interval $] 0, m[$ that appears at $\hat{D}=D$.

\section{B. Least-Mean Squares (LMS) Algorithm}

The minimization problem (7) can be solved with a LMS algorithm. The Least Mean Squares (LMS) algorithm [17] is an adaptive algorithm, which uses a gradient-based method of steepest decent. In the initialization phase, the user needs to provide an estimate $\hat{D}^{0}$ and a step size parameter $\mu$. At each step the LMS algorithm will adapt the estimate $\hat{D}$ according to (11). We have selected a normalized LMS algorithm to simplify the choice of $\mu$, with variable step size to speed up the convergence. The analytical derivative is too complicated for efficient computation. We have chosen to substitute it by a finite difference approximation. The adaptive estimation algorithm is summarized in Algorithm 1.

To summarize, the LMS-based technique we propose is a robust way to estimate the time-skew to our required precision. The main drawback of this method is that it requires a relatively high computational effort.

\section{Simulation Results}

In order to validate the theoretical framework introduced in this paper, the behavioral model of a homodyne transmitter was constructed. Our choice was guided by the flexibility, high level of integrability and good performance of the homodyne transmitter. The block diagram of our homodyne transmitter is depicted in Fig. 1.

The periodically nonuniform technique, on which the entire framework is based, requires an explicit simulation of each carrier cycle. However, to keep the computational effort reasonable, the simulations presented in this paper are based on behavioral-passband models. The simulations are carried out in Matlab. The test signal is composed by $10 \mathrm{MHz}$ QPSK symbols shaped by a square root raised cosine filter with a rolloff factor of $\alpha=0.5$ and translated to the carrier frequency $f_{c}=1 \mathrm{GHz}$.

The BP-TIADC architecture is composed of two 10-bits ADCs, each one operating at $B=1 / T=90 \mathrm{MHz}$. We consider that there are no gain or offset mismatches between the two ADCs. The clock generator that drives the sampleand-hold circuit is affected by a gaussian distributed time-skew jitter of 3 ps rms.

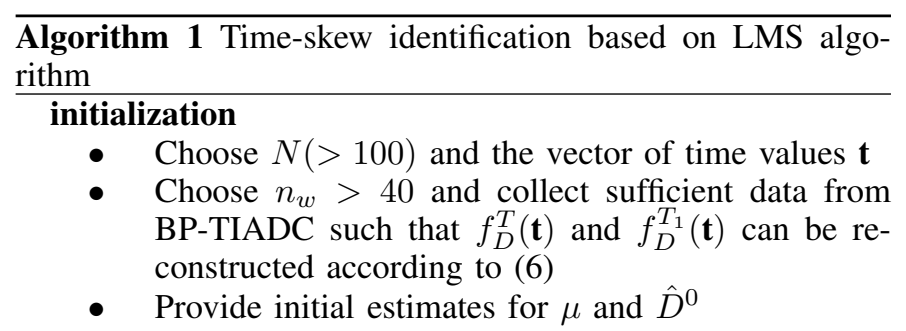

repeat

1. Calculate $f_{\hat{D}^{i}}^{T}(\mathbf{t})$ and $f_{\hat{D}^{i}}^{T_{1}}(\mathbf{t})$ using (6)

2. Calculate the gradient $\nabla\left(\varepsilon_{T_{1}, \hat{D}^{i}}^{T, \hat{D}^{i}}(\mathbf{t})\right)$ numerically:

$$
\nabla\left(\varepsilon_{T_{1}, \hat{D}^{i}}^{T, \hat{D}^{i}}(\mathbf{t})\right)=\frac{\varepsilon_{T_{1}, \hat{D}^{i}}^{T, \hat{D}^{i}}(\mathbf{t})-\varepsilon_{T_{1}, \hat{D}^{i-1}}^{T, \hat{D}^{i-1}}(\mathbf{t})}{\hat{D}^{i}-\hat{D}^{i-1}}
$$

3. Update the parameter estimates:.

$$
\hat{D}^{i+1}=\hat{D}^{i}-\mu \frac{\nabla\left(\varepsilon_{T_{1}, \hat{D}^{i}}^{T, \hat{D}^{i}}(\mathbf{t})\right)}{\max \left|\nabla\left(\varepsilon_{T_{1}, \hat{D}^{i}}^{T, \hat{D}^{i}}(\mathbf{t})\right)\right|}
$$

4. Evaluate $\varepsilon_{T_{1}, \hat{D}^{i+1}}^{T, \hat{D}^{i+1}}(\mathbf{t})$

5. if $\varepsilon_{T_{1}, \hat{D}^{i}}^{T, \hat{D}^{i}}(\mathbf{t})<\varepsilon_{T_{1}, \hat{D}^{i+1}}^{T, \hat{D}^{i+1}}(\mathbf{t})$ then

5.1. $\mu=\mu / 2$

5.2. $\quad$ go to Step 3

endif

6. $\mu=2 \mu$

7. $i=i+1$

until $i$ is greater than a maximum limit or $\varepsilon_{T_{1}, \hat{D}^{i}}^{T, \hat{D}^{i}}(\mathbf{t})$ is sufficient small

In order to apply the LMS algorithm, a second set of samples taken at $B_{1}=B / 2=45 \mathrm{MHz}$ are used. We fix the delay $D$ to $180 \mathrm{ps}$. For these values of $B, B_{1}, D$, and $f_{c}$, $m=483 \mathrm{ps}$ and the conditions given in (9) are met.

Fig. 5 plots the cost function $\varepsilon_{T_{1}, \hat{D}}^{T, \hat{D}}(\mathbf{t})$ versus several values of $\hat{D}$ in this interval. As expected, the cost function has only one minimum that appears when $\hat{D}=D$. The cost function was calculated using $N=300$ random time values from the interval [470 ns, $1700 \mathrm{~ns}$ ]. In all simulations the reconstruction filter has 61 taps $\left(n_{w}=60\right)$ and is windowed by a Kaiser window.

The adaptive LMS algorithm is then run for several values of $\hat{D}^{0}$ spaced between $\left.\hat{D}^{0} \in\right] 0 \mathrm{ps}, 480 \mathrm{ps}[$. The initial value for $\mu$ was chosen equal to 1e-12. Fig. 6 plots the evolution of cost function for several values of $\hat{D}_{0}$. The algorithm is able to accurately estimate $D$ and converges, every time, in less than 20 iterations.

Finally, we adapted and implemented a second time-skew identification technique proposed by [14]. This technique uses a known input sinusoid of frequency $\omega_{0}$. The estimates provided by the two techniques were used for nonuniform reconstruction (1) of the test signal. The results are summarized in Table I. Three metric of interest are presented: the absolute (second column) and the relative (third column) difference 


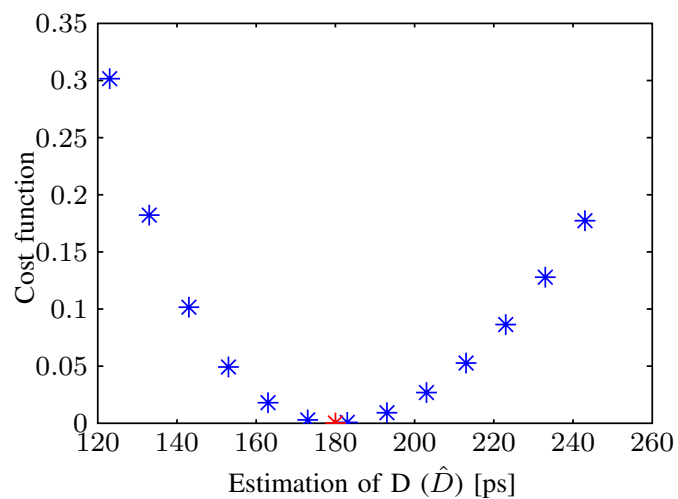

Fig. 5. Cost function for several values of $\hat{D}$

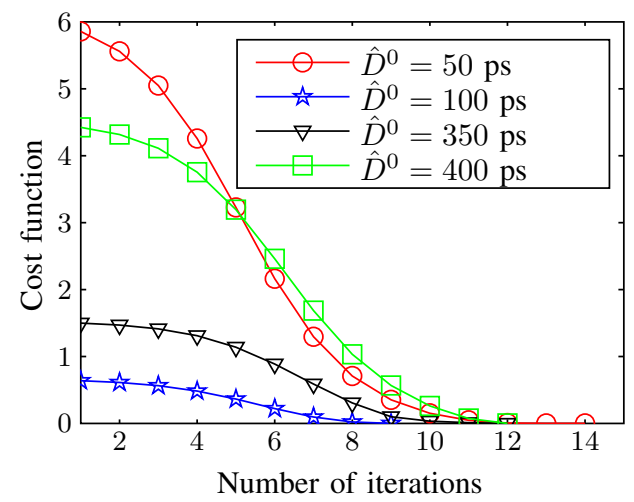

Fig. 6. LMS algorithm: evolution of cost function for several values of $\hat{D}_{0}$

between the real $D$ and its estimated value, and the relative error between the real signal and the reconstructed values (fourth column). The first two rows give the results obtained with the technique proposed by [14] for two values of $\omega_{0}$. The last two rows present the results of the LMS-based technique for two values of $\hat{D}_{0}$.

It can be seen that both techniques offer good estimates of $D$, but the first technique [14] is sensitive w.r.t. the frequency of the input test signal $\omega_{0}$. The LMS-based technique we propose performs better, doesn't need known test signal, and extracts the time-skew between two ADC in a robust way.

\section{CONCLUSions AND Future Works}

In this paper we introduced an RF BIST strategy based on Periodically Nonuniform Sampling of the signal at the output stages of multistandard radios. We leverage the I/Q ADC channels and the DSP resources to extract the bandpass waveform at the output of the power amplifier (PA). Analytical expressions and simulations show that our time-interleaved conversion scheme is sensitive to time-skew. We have evaluated a popular time-skew estimation approach [14], but found

TABLE I. TIME-SKEW ESTIMATION ANALYSIS

\begin{tabular}{lrrr}
\hline \hline & $|\hat{D}-D|$ & $|1-\hat{D} / D|$ & $\Delta \varepsilon\left(f_{\hat{D}}^{T}(t)\right)$ \\
\hline$\omega_{0}=0.4 B$ & $5 \mathrm{ps}$ & $2.8 \%$ & $3.5 \%$ \\
$\omega_{0}=0.46 B$ & $0.3 \mathrm{ps}$ & $0.1 \%$ & $1 \%$ \\
\hline$\hat{D}_{0}=50 \mathrm{ps}$ & $<0.1 \mathrm{ps}$ & $<0.1 \%$ & $0.84 \%$ \\
$\hat{D}_{0}=400 \mathrm{ps}$ & $<0.1 \mathrm{ps}$ & $<0.1 \%$ & $0.84 \%$ \\
\hline \hline
\end{tabular}

it restrictive and unreliable. We developed then an LMSbased time-skew estimation technique that is more accurate and robust. Using the delay estimates obtained, we were able to effectively reconstruct the output signal of the transmitter using our modified BP-TIADC architecture, opening the way for a complete RF BIST loopback strategy. Future developments will be focused on an efficient mapping to hardware of our nonuniform sampler.

\section{ACKNOWLEDGMENT}

This research has been supported by a CIFRE ANRT grant. The Supélec-Thales Chair on Advanced Analog Systems gratefully acknowledges the financial support of the Thales Group.

\section{REFERENCES}

[1] I. Mitola, J., "Software radios: Survey, critical evaluation and future directions," IEEE Trans. Aerosp. Electron. Syst., vol. 8, no. 4, pp. 2536, 1993.

[2] R. Wolf, M. Slamani, J. Ferrario, and J. Bhagat, Advances in Electronic Testing: Challenges and Methodologies. Netherlands: Springer, 2006, ch. 10 - RF Testing.

[3] P. Cruz, N. Carvalho, and K. Remley, "Designing and testing softwaredefined radios," IEEE Microw. Mag., vol. 11, no. 4, pp. 83-94, 2010.

[4] M. Negreiros, L. Carro, and A. A. Susin, "Reducing test time using an enhanced RF loopback," J. Electron. Testing, vol. 23, no. 6, pp. 613-623, 2007.

[5] J. Dabrowski and J. Bayon, "Mixed loopback BIST for RF digital transceivers," in Proc. IEEE Defect and Fault Tolerance in VLSI Systems (DFT 2004), Oct. 2004, pp. 220 - 228.

[6] A. Nassery and S. Ozev, "An analytical technique for characterization of transceiver IQ imbalances in the loop-back mode," in Design, Automation Test in Europe (DATE), 2012, pp. 1084-1089.

[7] A. Haldes, S. Bhattacharya, G. Srinivasan, and A. Chaterjee, "A systemlevel alternate test approach for specification test of RF transceivers in loopback mode," in Proc. Int. Conf. on VLSI Design (VLSID'05), 2005, pp. 132-137.

[8] Y.-P. Lin and P. Vaidyanathan, "Periodically nonuniform sampling of a new class of bandpass signals," in Proc. IEEE Digital Signal Processing Workshop, Sep. 1996, pp. $141-144$.

[9] A. Kohlenberg, "Exact interpolation of band limited functions," J. Appl. Physics, vol. 24, no. 12, pp. 1432 -1436, Dec. 1953.

[10] B. Razavi, RF microelectronics. Prentice Hall PTR, 1998.

[11] P. Cruz and N. B. Carvalho, Advanced Microwave and Millimeter Wave Technologies Semiconductor Devices Circuits and Systems. Austria: In-Tech, 2010, ch. 25 - Receiver Front-End Architectures - Analysis and Evaluation.

[12] R. Vaughan, N. Scott, and D. White, "The theory of bandpass sampling," IEEE Trans. Signal Process., vol. 39, no. 9, pp. 1973-1984, Sep. 1991.

[13] D. Camarero, K. Ben Kalaia, J.-F. Naviner, and P. Loumeau, "Mixedsignal clock-skew calibration technique for time-interleaved adcs," IEEE Trans. Circuits Syst. I, vol. 55, no. 11, pp. 3676 -3687, Dec. 2008.

[14] S. Jamal, D. Fu, M. Singh, P. Hurst, and S. Lewis, "Calibration of sample-time error in a two-channel time-interleaved analog-to-digital converter," IEEE Trans. Circuits Syst. I, vol. 51, no. 1, pp. 130 - 139, Jan. 2004.

[15] V. Arkesteijn, E. A. M. Klumperink, and B. Nauta, "Jitter requirements of the sampling clock in software radio receivers," IEEE Trans. Circuits Syst. II, vol. 53, no. 2, pp. 90-94, 2006.

[16] D. Fu, K. Dyer, S. Lewis, and P. Hurst, "A digital background calibration technique for time-interleaved analog-to-digital converters," IEEE J. Solid-State Circuits, vol. 33, no. 12, pp. 1904 -1911, Dec. 1998.

[17] S. Haykin, Adaptive filter theory. Upper Saddle River, N.J: Prentice Hall, 2002. 